\title{
Phage-derived lysins as therapeutic agents against multidrug-resistant Enterococcus faecalis
}

\author{
Jingmin $\mathrm{Gu}^{*}{ }^{1}$, Hengyu $\mathrm{Xi}^{1}$, Mengjun Cheng ${ }^{1}$ \& Wenyu Han**,1,2 \\ ${ }^{1}$ College of Veterinary Medicine, Jilin University, Changchun 130062, PR China \\ ${ }_{2}^{2}$ Jiangsu Co-innovation Center for the Prevention \& Control of important Animal Infectious Disease \& Zoonoses, Yangzhou \\ 225009, PR China \\ * Author for correspondence: Tel./Fax: +86 4318783 6406; jingmin0629@163.com \\ ** Author for correspondence: hanwy@jlu.edu.cn
}

\section{"Our enemy's enemy is our friend. Thus, we can borrow the weapons of our friends to fight common enemies."}

First draft submitted: 18 October 2017; Accepted for publication: 20 October 2017; Published online: 14 February 2018

Keywords: Enterococcus faecalis • lysin $\bullet$ multidrug resistant $\bullet$ opportunistic pathogen $\bullet$ phage

Enterococcus faecalis is a Gram-positive bacteria that is commonly a part of the normal microflora in the oral cavity, lower intestinal tract and vaginal tract of animals and people [1]. However, it is also an opportunistic pathogen that can become invasive, causing nosocomial infections and even life-threatening infections in immunocompromized patients [2]. The high prevalence of multidrug-resistant $E$. faecalis, especially vancomycin-resistant $E$. faecalis (VREF), has made these infections extremely difficult to eradicate using conventional antibiotics and it has thus intensified the threat to global health [3]. However, therapeutically available options to control multidrug-resistant $E$. faecalis infections are very limited at present. Thus, new therapeutic agents for this opportunistic pathogen are urgently needed.

Bacteriophages (phages) are bacterial viruses, which are also called the 'natural killers' of bacteria. Phages can hijack their bacterial hosts for their propagation. The effectiveness of phage therapy has been established by virtue of the phages' ability to kill target pathogens. Since its establishment in 1923, the Eliava Institute of Bacteriophages in Georgia has supplied phage therapeutics for treating various bacterial infections [4]. Phage therapy is seen as a reliable treatment option when antibiotics are ineffective against bacterial infections. Tom Patterson's recent narrow escape from the fatal infection caused by multidrug-resistant Acinetobacter baumannii proved the effectiveness of phage therapy.

Endolysin (lysin) therapy is the main part of phage therapy. Lysins encoded by phages are highly evolved peptidoglycan hydrolases that lyse the host cell at the terminal stage of the phage lytic lifecycle to ensure the release of phage progeny [5]. Phage-derived lysins have great therapeutic potential due to their capacity to induce immediate lysis of the target bacterium when added externally. The rising interest in lysin therapy occurred due to the increasing prevalence of bacterial antibiotic resistance and the growing need for novel classes of antibacterial agents. Several studies have shown that recombinant phage-derived lysins exhibit highly efficient bactericidal activity against multidrug-resistant $E$. faecalis both in vitro and in mice models. Considering the special role of $E$. faecalis in animals and people, treatment of this opportunistic pathogen with lysins has several advantages.

\section{High efficiency}

Phage-derived lysins are highly efficient enzymes that directly attack one of four major peptidoglycan bonds and thus degrade the peptidoglycan layer of bacterial cell walls. The cell wall is responsible for maintaining the bacterial shape and withstanding internal osmotic pressure. Generally, exogenous administration of lysin promotes bacterial osmotic lysis by forming holes in the cell wall within seconds of contact, ultimately resulting in the death of the target Gram-positive bacterium, much faster than most antibiotics [6]. Several studies have reported that lysins are

Future Medicine 
highly efficient in terms of lysing E. faecalis, including LysEF-P10 [7], the endolysin of IME-EF1 [8], the lysin of ФEF24C [9], PlyV12 [10], Lys168 and Lys170 [11] and the lysin of VD13 [12]. In particular, LysEF-P10 (20 $\mu \mathrm{g} / \mathrm{ml})$ exhibited extremely efficient bactericidal activity, with the viable $E$. faecalis count decreasing from $10^{8} \mathrm{CFU} / \mathrm{ml}$ by approximately $4 \log$ units at $10 \mathrm{~min}$ after treatment and by approximately $7 \log$ units at $60 \mathrm{~min}$. More importantly, a single administration of LysEF-P10 $(5 \mu \mathrm{g})$ was sufficient to protect mice against lethal VREF infection [7].

It should be mentioned that the bactericidal effects of these lysins are not affected by the presence of antibiotic resistance mechanisms among the bacteria. Thus, multidrug-resistant strains, including VREF, are also sensitive to lysins. The effects are attributed to the unique bactericidal mechanism of lysins, which is different from that of antibiotics.

\section{Lack of resistance}

Usually, most antibiotics inhibit an essential bacterial metabolic step, which leads to the slow deterioration of cellular conditions and, finally, cell death [13]. However, there are many alternative metabolic pathways in bacteria. When one of the pathways is blocked, other pathways are able to replace it. Thus, antibiotic resistance develops very easily. This situation does not apply to phage-derived lysins. Despite several attempts to develop lysin-resistant strains by repeated lysin exposure, no cases of such resistance mutations have been reported to date [14]. Recently, we proved that LysEF-P10-resistant E. faecalis strains were not generated after repeated exposure to a sublethal concentration of LysEF-P10 both on agar plates and in liquid culture [UNPUBLISHED DATA]. This may be attributable to the mode of action and target of lysins, as the bactericidal activity of lysins is independent of active host metabolism.

The enzymatic catalysis mediated by lysins is direct and fast compared with the mode of action of antibiotics. In addition, the co-evolution over billions of years between bacteria and their phages has refined lysins with excellent properties $[13,14]$. Both the binding and catalytic domains of lysins target the components of the bacterial peptidoglycan skeleton structure, which is generally species specific and critical for bacterial viability. Furthermore, the damage is typically difficult for the bacteria to reverse, making the development of resistance to lysins a rare event [15].

\section{Lack of neutralizing antibodies}

The molecular weight of most phage-derived lysins is $>20 \mathrm{kDa}$. Thus, lysins can be immunogenic. Indeed, $E$. faecalis LysEF-P10 [7] and the phage-derived lysins that target other bacteria [5] have been shown to induce the formation of antibodies. Very interestingly, the specific antibodies only slow but do not eradicate the lytic activity of lysins. Most importantly, the rescue of LysEF-P10-immunized E. faecalis-infected mice using LysEF-P10 treatment was equal to that of nonimmunized $E$. faecalis-infected mice, with no side effects.

However, it is unclear why the specific antibodies do not eliminate the bactericidal activity of the lysins. This phenomenon may be explained by two hypotheses. First, the antibodies induced by lysins are not neutralizing antibodies [16]. The antibodies do not block the key activity-related sites of the binding and catalytic domains of the lysins. The key amino acids responsible for binding and catalysis are usually located in the groove that contains many hydrophobic amino acids. Second, the affinity of lysins to their bacterial targets is much higher than that of the antibodies for the lysins [17]. The binding and catalytic targets of lysins are the products of long-term evolution and so they result in more stable binding.

\section{Specificity}

In general, phage-derived lysins exhibit broader bactericidal activity than the phages they are derived from. However, the bactericidal spectrum of lysins is much narrower than that of antibiotics, which usually exhibit broad-spectrum activity, eliminating many different bacteria colonizing the body, including members of the normal microflora.

Lysin-binding domains bind a specific ligand in the cell wall with high affinity, resulting in high specificity. In most instances, lysins are specific to a single bacterial species, only killing that species. For example, LysEF-P10 and the lysin of VD13 can only lyse E. faecalis strains [7,12]. The high specificity of these lysins guarantees (as far as possible) the elimination of the target pathogen, without overly affecting the commensal microflora, including Enterococcus faecium. Enterococcus faecalis strains are usually considered harmless commensal strains in healthy humans and animals. The prophylactic administration of lysins most likely causes suppression of the normal $E$. faecalis in the body, which then affects the commensal microflora. Our study showed that the genus Enterococcus in the intestinal microflora was reduced after treatment with $5 \mu \mathrm{g}$ LysEF-P10 [7]. Thus, treatment using lysins is feasible, but prophylactic administration is not. 
On the other hand, some lysins exhibit a broad lytic spectrum, killing many different bacterial species. Several lysins have been reported not only to lyse E. faecalis, but also to kill E. faecium isolates, including the lysin of IME-EF1 [8], the lysin of $\Phi E F 24 C$ [9], PlyV12 [10], Lys168 and Lys170 [11] and the lysin of EFAL-1 [18]. These lysins may also kill normal E. faecium in the body when eliminating the E. faecalis infection. Nevertheless, these lysins can be used as decontaminants to reduce the environmental load of E. faecalis and E. faecium in areas in which these bacteria are a serious problem, such as in hospitals.

\section{Areas for further investigation}

Before lysins are widely used as therapeutics or biocontrol agents to treat clinical E. faecalis infections, five key research areas need to be addressed. Phages are the most abundant biological entity on Earth $\left(10^{31}-10^{32}\right)$ and phage-derived lysins exhibit a high degree of biochemical diversity [19], more E. faecalis phage-derived lysins should be identified. To identify the mechanism underlying their efficient lytic activity, the three-dimensional structures of $E$. faecalis phage-derived lysins and particularly the structure of the lysin-target molecule complex, need to be resolved. Any synergy between $E$. faecalis phage-derived lysins that cleave different peptidoglycan bonds needs to be identified. More information on the optimization of the dosing regime, pharmacokinetics and pharmacodynamics of using lysins to treat E. faecalis infection in vivo is crucial. Well-designed human clinical trials to examine the efficiency of lysins for treating $E$. faecalis infection remain critical.

\section{Conclusion}

Our enemy's enemy is our friend. Thus, we can borrow the weapons of our friends to fight common enemies. In summary the high efficiency, lack of resistance, lack of neutralizing antibodies and specificity make phage-derived lysins a great therapeutic agent against multidrug-resistant $E$. faecalis.

\section{Financial \& competing interests disclosure}

J Gu and W Han are financially supported through grants from the National Natural Science Foundation of China (grant numbers 31572553 and 31502103). The authors have no other relevant affiliations or financial involvement with any organization or entity with a financial interest in or financial conflict with the subject matter or materials discussed in the manuscript apart from those disclosed.

No writing assistance was utilized in the production of this manuscript.

\section{References}

1 Koch S, Hufnagel M, Theilacker C, Huebner J. Enterococcal infections: host response, therapeutic, and prophylactic possibilities. Vaccine 22(7), 822-830 (2004).

2 Cetinkaya Y, Falk P, Mayhall CG. Vancomycin-resistant enterococci. Clin. Microbiol. Rev. 13(4), 686-707 (2000).

3 Van Harten RM, Willems RJL, Martin NI, Hendrickx APA. Multidrug-resistant enterococcal infections: new compounds, novel antimicrobial therapies? Trends Microbiol. 25(6), 467-479 (2017).

4 O'Flaherty S, Ross RP, Coffey A. Bacteriophage and their lysins for elimination of infectious bacteria. FEMS Microbiol. Rev. 33(4), 801-819 (2009).

5 Fischetti VA. Bacteriophage lysins as effective antibacterials. Curr. Opin. Microbiol. 11(5), 393-400 (2008).

6 Schmelcher M, Donovan DM, Loessner MJ. Bacteriophage endolysins as novel antimicrobials. Future Microbiol. 7(10), 1147-1171 (2012).

7 Cheng M, Zhang Y, Li X et al. Endolysin LysEF-P10 shows potential as an alternative treatment strategy for multidrug-resistant Enterococcus faecalis infections. Sci. Rep. 7(1), 10164 (2017).

8 Zhang W, Mi Z, Yin X et al. Characterization of Enterococcus faecalis phage IME-EF1 and its endolysin. PLoS ONE 8(11), e80435 (2013).

9 Uchiyama J, Takemura I, Hayashi I et al. Characterization of lytic enzyme open reading frame 9 (ORF9) derived from Enterococcus faecalis bacteriophage phiEF24C. Appl. Environ. Microbiol. 77(2), 580-585 (2011).

10 Yoong P, Schuch R, Nelson D, Fischetti VA. Identification of a broadly active phage lytic enzyme with lethal activity against antibiotic-resistant Enterococcus faecalis and Enterococcus faecium. J. Bacteriol. 186(14), 4808-4812 (2004).

11 Fernandes S, Proenca D, Cantante C et al. Novel chimerical endolysins with broad antimicrobial activity against methicillin-resistant Staphylococcus aureus. Microb. Drug Resist. 18(3), 333-343 (2012).

12 Swift SM, Rowley DT, Young C, Franks A, Hyman P, Donovan DM. The endolysin from the Enterococcus faecalis bacteriophage VD13 and conditions stimulating its lytic activity. FEMS Microbiol. Lett. 363(19), pii:fnw216 (2016). 
13 Gerstmans H, Rodriguez-Rubio L, Lavigne R, Briers Y. From endolysins to Artilysin(R)s: novel enzyme-based approaches to kill drug-resistant bacteria. Biochem. Soc. Trans. 44(1), 123-128 (2016).

14 Rios AC, Moutinho CG, Pinto FC et al. Alternatives to overcoming bacterial resistances: state-of-the-art. Microbiol. Res. 191, 51-80 (2016).

15 Muller S, Wolf AJ, Iliev ID, Berg BL, Underhill DM, Liu GY. Poorly cross-linked peptidoglycan in MRSA due to mecA induction activates the inflammasome and exacerbates immunopathology. Cell Host Microbe 18(5), 604-612 (2015).

16 Zhang L, Li D, Li X et al. LysGH15 kills Staphylococcus aureus without being affected by the humoral immune response or inducing inflammation. Sci. Rep. 6, 29344 (2016).

17 Loessner MJ, Kramer K, Ebel F, Scherer S. C-terminal domains of Listeria monocytogenes bacteriophage murein hydrolases determine specific recognition and high-affinity binding to bacterial cell wall carbohydrates. Mol. Microbiol. 44(2), 335-349 (2002).

18 Son JS, Jun SY, Kim EB et al. Complete genome sequence of a newly isolated lytic bacteriophage, EFAP-1 of Enterococcus faecalis, and antibacterial activity of its endolysin EFAL-1. J. Appl. Microbiol. 108(5), 1769-1779 (2010).

19 Suttle CA. Marine viruses - major players in the global ecosystem. Nat. Rev. Microbiol. 5(10), 801-812 (2007). 